\title{
CARDIOVASCULAR SYSTEM OF THE MATURE RATS WITH CONGENITAL HYPOTHYROIDISM AND ARTERIAL HYPERTENSION
}

10.36740/WLek202010119

\author{
Svetlana N. Chuhray' ${ }^{1}$, Viktoria E. Lavrynenko ${ }^{2}$, Rostyslav F. Kaminsky' ${ }^{1}$ Larysa B. Shobat ${ }^{1}$, Oleksandr I. Kovalchuk ${ }^{2}$, \\ Yuri B. Chaikovsky ${ }^{1}$, Liudmyla M. Sokurenko ${ }^{1,2}$ \\ 'BOGOMOLETS NATIONAL MEDICAL UNIVERSITY, KYIV, UKRAINE \\ ${ }^{2}$ TARAS SHEVCHENKO NATIONAL UNIVERSITY OF KYIV, KYIV, UKRAINE
}

\begin{abstract}
The aim: Studying changes in the ultrastructure of blood circulatory capillaries of the myocardium of mature rats with hypothyroidism and arterial hypertension. Materials and methods: Experiments were conducted on (240 days) 10 ISIAH (inherited stress-induced arterial hypertension) line rats with AH (arterial hypertension), 10 Wistar line rats with congenital hypothyroidism and 10 intact animals. Arterial pressure was measured, and the development of hypothyroidism was controlled by the immune enzyme method. The study of the left ventricle myocardium of the rat heart was carried out by electron microscopic and morphometric studies.

Results: In in rats with AH the following changes were observed in the blood capillaries of the myocardium: decrease in the number of capillaries; disturbance of blood circulation; the number of organelles of the biosynthetic plan and structures involved in the transendothelial transfer of substances decreased in endothelial cells; lysis and edema of the latter; mucinous perivascular edema, confirmed by the accumulation of fine-fibrillar structures, collagen fibers, cellular detritus. By the same term, in the group with congenital hypothyroidism, dystrophic-destructive changes in the blood capillaries of the myocardium acquired the highest degree, which resulted in a decrease in their number due to destruction. Ultrastructure of the biosynthetic plan organelles and structures of the transendothelial transfer of substances were in decompensated state.

Conclusions: The rats (in 240 days) with AH and congenital hypothyroidism express breakdown of compensatory processes in the capillaries of the myocardium. This is manifested by the further dilution of capillaries, the development of hypoxic state in them as well as mucinous edema of interstitium, the decrease of activity of biosynthetic and transport processes.
\end{abstract}

KEY WORDS: myocardium, blood capillaries, congenital hypothyroidism, hypertension, electron microscopy

Wiad Lek. 2020;73(10):2209-2213

\section{INTRODUCTION}

Arterial hypertension is a pathological condition that is accompanied by a constant or periodic increase in blood pressure in comparison with the age norm. According to statistics, one fifth of the adult population of the world suffers from arterial hypertension [1].

In most cases, arterial hypertension is secondary to diseases of the kidneys, cardiovascular, endocrine conditions. Among the latter a significant place belongs to hypothyroidism, a disease caused by a deficiency of hormones of the thyroid gland in the body, or a decrease in their biological effect at the tissue level [2]. Arterial hypertension is found in $10-30 \%$ of patients with hypothyroidism, and disorders of hemomicrocirculation play an important, and sometimes crucial, role in its pathogenesis $[3,4,5]$. Since there is evidence of the difference between the clinical manifestations of hypothyroidism and arterial hypertension, depending on age $[6,7]$ we were interested in the difference in morphofunctional changes in these diseases.

\section{THE AIM}

The study of changes in the ultrastructure of blood circulatory capillaries of the myocardium of mature rats with hypothyroidism and arterial hypertension.

\section{MATERIALS AND METHODS}

Experiments were carried out on 30 white rats, which were under standard vivarium conditions of the Bogomolets National Medical University. The adult 240-day rats of the ISIAH line (10 rats) and the Wistar rats with congenital hypothyroidism (10 rats) were studied. Control was provided by intact rats (10 rats) of the Wistar line of the same age.

Pregnant female rats were administered with thyreostatic drug - Mercazolil beginning from the $14^{\text {th }}$ day of perinatal development, in order to simulate congenital hypothyroidism by inhibiting functioning of thyroid gland. Mercazolil was received by animals with mother milk right after birth and with self-feeding later. Throughout the study the daily dose of the thyreostatic drug was $20 \mathrm{mg} / \mathrm{kg}$ of body weight. Water was used as a solvent. The immune-enzymatic method on the Sunrise RC device, TEKAN was used to control the development of hypothyroidism in both control and hypothyroidic rats after removing from the experiment.

Arterial hypertension was studied on ISIAH (inherited stress-induced arterial hypertension) line rats with $\mathrm{AH}$ (arterial hypertension) which serve as a model of human hypertensive disease $[8,9,10]$. Experiments with animals were carried out in accordance with the Law of Ukraine "On 
Table I. Changes of arterial pressure figures

\begin{tabular}{cccc}
\hline & \multicolumn{3}{c}{ 240 days } \\
\cline { 2 - 4 } & Control & AH & CHT \\
\hline $\begin{array}{c}\text { Arterial pressure, } \\
\mathrm{mm} \mathrm{Hg}\end{array}$ & $117,6 \pm 4,2$ & $151,2 \pm 5,3^{*}$ & $136,2 \pm 4,3^{*},{ }^{* *}$ \\
\hline
\end{tabular}

Note: ${ }^{*}-p<0,05$ in comparison with the data of the Control;

${ }^{* *}-p<0,05$ in comparison with the data of group with the other model.

protection of animals from cruelty" (2006), "General ethical principles of animal experiments", adopted by the First National Congress on Bioethics (Kiev, 2001) and in accordance with the requirements of the "European Convention for the Protection of Vertebrate Animals Used for Experimental and other Scientific Purposes" (Strasbourg, 1985). Measuring of arterial pressure, morphometric studies, electron microscopic examination were conducted as described in the previous article on study of cardio-vascular system $[11,12,13,14]$.

Plethysmograph was used to measure AP (arterial pressure) of rats from all experimental groups when they

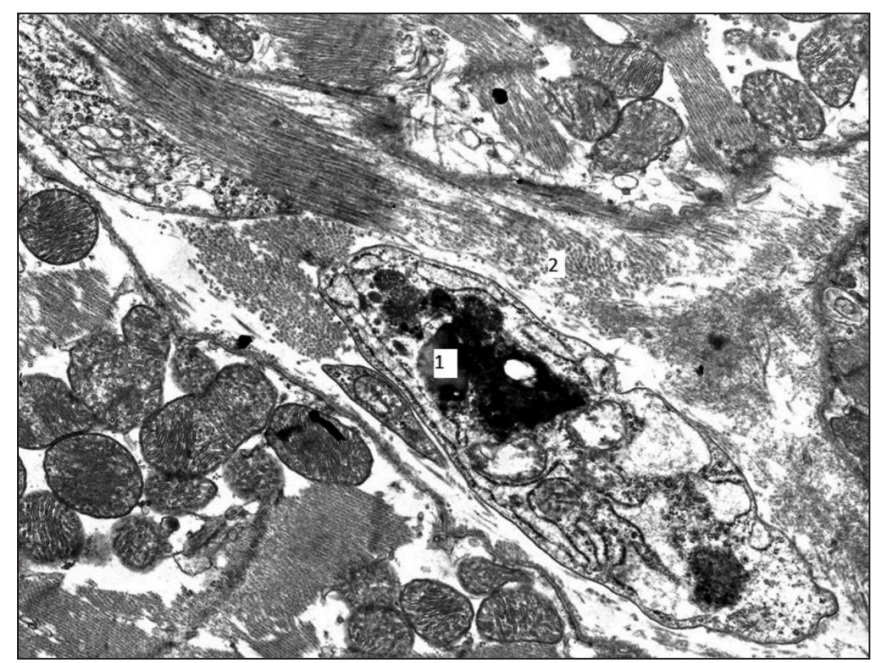

Fig.1. Blood capillary of left ventricle of myocardium in 240-day-old rat with AH. Electron microscopic photo. Magn.: 12000. Macrophage (1), collagen fibers (2) in the perivascular space.

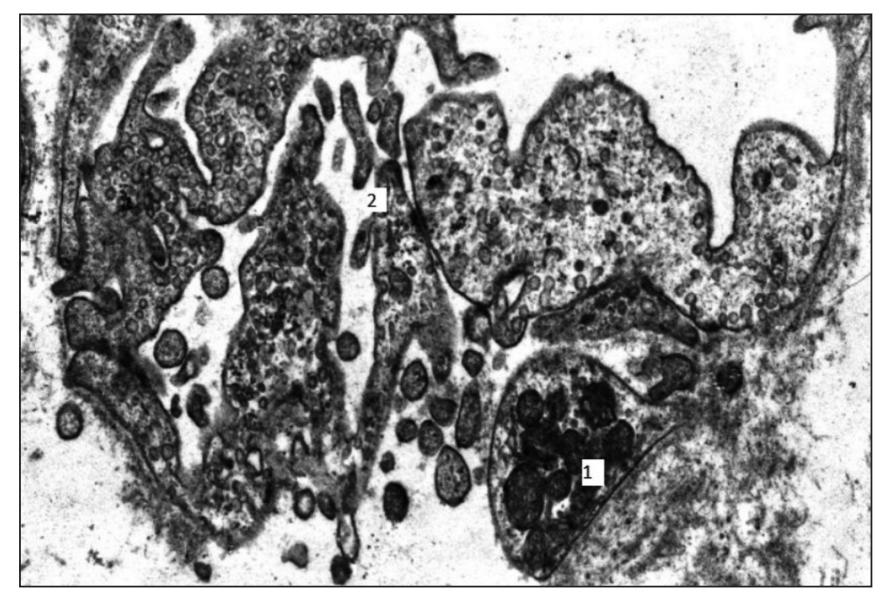

Fig.2. Blood capillaries of the left ventricle myocardium in 240-day rat with CHT. Microclasmatic outgrowths (1) in endothelial cells (2). Electron microscopic photo. Magn.: 22000. were removed from the experiment. The left ventricular myocardium of rats from the experimental group was studied using electron microscopy. The commonly accepted techniques of electron microscopic study were used to process the material [15]. Ultratome Reihart (Austria) was used to make ultrathin sections which were later studied with electron microscope PEM-125K.

The application "Organelle" was used to process the data of morphometric studies, which were conducted on a semi-automatic device for graphic research. Micropinocytotic vesicles (MPV) were analyzed using such characteristics as cross-section area, volumetric and quantitative densities and form factor. The Student parametric and the Kolmogorov-Smirnov nonparametric criterions were used for statistical processing of all received data according to the variation statistics principle.

\section{RESULTS}

Arterial pressure in 240-day-old rats with arterial hypertension was $151.2 \pm 5.3 \mathrm{~mm} \mathrm{Hg}$ while in rats with congenital hypothyroidism $-136,2 \pm 4,3 \mathrm{~mm} \mathrm{Hg}$ that is statistically significantly more than in intact rats $-117,6 \pm 4,2 \mathrm{mmHg}$ (Table I). At the same time, in rats with arterial hypertension, the pressure was significantly higher in each animal. 240-day-old rats with arterial hypertension showed domination of blood capillaries with somewhat diminished lumen diameter comparing to age-control. The walls of some capillaries formed folds, which may be the result of increased contractility of cardiomyocytes, and as a result - a decrease in the lumen of the vessels. As a rule, accumulations of formed elements of blood were found in these lumens, that significantly impaired the circulation of blood.

The internal contour of hemocapillaries was fairly even due to the absence of microvilli and invaginations on their luminal surface (Fig. 1). In blood capillaries, endothelial cells of slightly elevated electron density alternated with electron-transparent cells (Fig. 1).

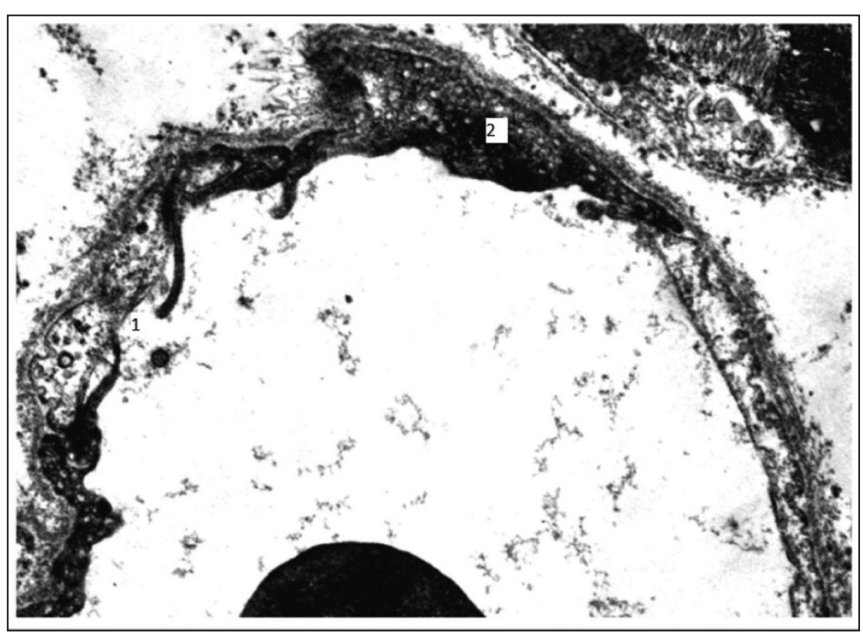

Fig.3. Blood capillaries of the left ventricle myocardium in 240-day rat with CHT. Electron microscopic photo. Magn.: 23000. Light (1) and dark (2) endotheliocytes. 
Table II. The morphometric indexes of micropinocytotic vesicles in endothelial cells of myocardial circulatory capillaries

\begin{tabular}{cccc}
\hline Indexes & Control & Rats with AH & Rats with CHT \\
\hline Volumetric density, $\%$ & $16,81 \pm 2,16$ & $9,66 \pm 1,01^{*}$ & $10,54 \pm 1,12^{*}$ \\
\hline Quantitative density, $1 / \mu m^{3}$ & $335,3 \pm 71,7$ & $56,5 \pm 7,2^{*}$ & $69,3 \pm 8,3^{*}$ \\
\hline Average area, $10^{-2} \mu \mathrm{m}^{2}$ & $0,52 \pm 0,02$ & $1,17 \pm 0,03^{*}$ & $0,97 \pm 0,02^{*}{ }^{* *}$ \\
\hline Form factor & $0,78 \pm 0,01$ & $0,83 \pm 0,01^{*}$ & $0,85 \pm 0,01^{*}$ \\
\hline
\end{tabular}

Note: ${ }^{*}-p<0,05$ in comparison with the data of the Control;

$*^{*}-p<0,05$ in comparison with the data of group with the other model.

The former, as in the age control, had thinned peripheral areas, and the increase in electron density occured due to the accumulation of the electron-dense material in the cytoplasm. Electron-transparent cells had a wider endothelium and reflected different stages of lysis and edema of cells. Increased number of edematous endothelial cells indicated deterioration of hemomicrocirculation.

The nuclei of both types of endothelial cells had an elongated, sometimes irregular shape. Perinuclear space in general was not extended. Euchromatin was evenly distributed around the karyoplasm.

In comparison with age-control, the number of elements of the rough endoplasmic reticulum and ribosomes in the cytoplasm of endothelial cells decreased, there were almost no secretory vesicles. Swollen mitochondria of different size, oval and rounded shape with lysed cristae and unevenly cleared matrix were observed. Endothelial cells were interconnected with junctions of different length, where the open areas alternated with dense, so-called, obliteration spots. Basal membranes of capillaries were expanded due to the dissolution of their fibrillar components. Dense bundles of collagen fibers and immune-competent cells, in particular, macrophages, accumulated in the extended perivascular spaces.

In the myocardium of 240-day rats with congenital hypothyroidism, the number of blood capillaries was significantly reduced compared with age control. Endothelial cells in these microvessels were altered: some of them changed in the dark type, others - in the light, and they alternated with each other. There were also endothelial cells, the cytoplasm of which had both electron-dense and electron-transparent portions. The lumen surface of the latter and of the dark endothelial cells formed deep invaginations and outgrowths, some of which became microclasmatic and desquamated into the lumen (Fig. 2). Electron-dense areas were formed by finely dispersed material, which lacked organelles. Single mitochondria, as a rule, had a lysed matrix and cristae. Nuclei of such cells had deep invaginations; the karyoplasm had a spotty appearance.

Electron-transparent endothelial cells had an insignificant thickness of peripheral regions, mostly devoided of organelles, with the exception of the micropinocytotic vesicles. The latter were also observed in some cells (Fig.3.). Given that these endothelial cells were not dilated, we can assume that enlightenment of the cytoplasm was associated with its lysis, and edema was not inherent to them.

Morphometric analysis of micropinocytosic vesicles in rats at 240 days showed a significant increase in the average area of these vesicles, compared with age control. It happened, most likely, due to the merging of some of them, as well as prevailing processes of inhibition of its formation. Micropinocytotic vesicles, as in the control, were located on both basal and luminal surfaces, and freely around the cytoplasm. Morphometric analysis showed their significant quantitative changes (Table 2).

Thus, the volumetric density of the micropinocytotic vesicles in the endothelial cells of rats with arterial hypertension was $9.66 \pm 1.01 \%$, which is statistically significantly less than that of normotensive rats $(16.81 \pm 2.16 \%)$, and even less than in animals with congenital hypothyroidism (10.54 \pm $1.12 \%)$. In contrast to the index of intact rats $(335.3 \pm$ 71.7 per $1 \mu^{3}$ ), their quantitative density decreased almost 6 times, which was $56.5 \pm 7.2$ per $1 \mu \mathrm{m}^{3}$, in rats with congenital hypothyroidism this index was $69.3 \pm 8.3$ per 1 $\mu^{3}$. The average area of the cross-section of micropinocytotic vesicles in the endothelial cells of hypertensive rats statistically significantly increased almost twice to $1.17 \pm$ $3.610^{-2} \mu \mathrm{m}^{2}$ comparing to intact rats $\left(0.52 \pm 0.02^{-2} \mu \mathrm{m}^{2}\right)$ and rats with CHT $\left(0.97 \pm 0.0210^{-2} \mu \mathrm{m}^{2}\right)$. They became more rounded as evidenced by the index of the form factor $(0.83 \pm 0.01)$, which became closer to 1,00 . An increase in the area of the vesicles may be due to the fact that vacuolar formations participated in the transfer of substances from lumen to interstitium and vice versa. The form factor of micropinocytotic vesicles in endothelial cells in rats with congenital hypothyroidism was $0,85 \pm 0,01$, that is, they also became more rounded.

\section{DISCUSSION}

In 240-day-old rats with spontaneous arterial hypertension, and with congenital hypothyroidism, an impairment of compensatory processes in the blood capillaries of the myocardium were marked. The nature of the changes in both experimental groups had common features and differences.

The common features included: firstly, a decrease in the number of blood capillaries; and secondly, circulation disturbance as well as blood and ultrastructural signs of hypoxic condition, as evidenced by the presence of erythrocytes, platelets and fine-dispersed material in the lumen of the capillaries; thirdly, a significant decrease in the functional activity of endothelial cells, a sign of which is a decrease in the number of organelles of the biosynthetic plan (mitochondria, polysomes, endoplasmic 
reticulum, Golgi complex) and structures involved in the transendothelial transfer of substances - micropinocytotic vesicles; fourthly, lysis and edema of endothelial cells; fifth, perivascular edema, which is mucinous, that is confirmed by the accumulation of fine-fibrous structures, which are likely proteoglycans and glycoproteins, collagen fibers, cellular detritus. Morphological signs of a hypoxic state are explained by various pathogenic mechanisms [16], oxidative stress may lead to reduced bioavailability of nitric oxide [17], increased production of endothelin-1 [18], elevated intracellular $\mathrm{Ca}^{2+}$ [19], vascular remodeling [20] or renal pathologies [21], which directly or indirectly promote vasoconstriction and blood pressure elevation [22]. Therefore, it is worth pursuing in the future studies to investigate the impact of hypothyroidism-like mechanisms of hypertensive conditions.

As known, Triiodothyronine affects the hemodynamic state mainly by its influence on the expression of cardiomyocyte genes, which encode both structural and regulatory proteins in the heart (myosin heavy chains, sarcoplasmic reticulum calcium-activated ATP-ase, phospholamban) and other intracellular mechanisms of thyroid hormone action in the cardiomyocytes [23].

The main differences in the ultrastructural organization of myocardial blood capillaries in 240-day-old rats with hypertension and rats with congenital hypothyroidism were more prevalent and expressive destructive-dystrophic changes in the latter as well as the presence of endothelial cells that are at different stages of apoptosis. Such differences are due, apparently, to the fact that in rats with congenital hypothyroidism, arterial hypertension develops against the background of deficiency of thyroid hormones and calcitonin. The fact that hyperthyroid rats develop apoptotic altered endothelial cells during the development of hypothyroidism is another indication that the main trigger mechanism for apoptosis is the receptor pathway. That is, in rats with uncorrected congenital hypothyroidism, the death of endothelial cells occurs in two ways - apoptosis and necrosis. Thus, thyroid hormone changed cardiovascular hemodynamics.

The literature informs that the aging is associated with alterations in the hypothalamic-pituitary-thyroidal axis which can lead to hypothyroidism. Increase in the percentages of large follicles and significant decrease in FT3 level was observed in 18-month-old rats in comparison to 3-month ones [21].

\section{CONCLUSIONS}

In rats of mature age (240 days) with arterial hypertension and congenital hypothyroidism there is a breakdown of compensatory processes in the capillaries of the myocardium. This is manifested by the further dilution of capillaries, the development of hypoxic state and mucinous edema of interstitium in them, the decrease of activity of biosynthetic and transport processes. At the same time, the expressiveness and prevalence of these processes, as in all previous periods of observation, is greater in rats with congenital hypothyroidism than in rats with arterial hypertension.

\section{REFERENCES}

1. Chobanian A., BakrisG.,BlackH. etal.TheSeventh Report of the Joint National Committee on Prevention, Detection, Evaluation, and Treatment of High Blood Pressure.JAMA. 2003;289:2560-72. D01: 10.1001/jama.289.19.2560

2. Antonenko A., Vavrinevych 0., Korshun M., Omelchuk S. Hygienic assessment of the effects of pesticides application on children population morbidity with thyroid gland diseases Wiad Lek. 2019; 72(2): 267-70.

3. Lutai M., Slobodskoy V. Endothelial dysfunction with ischemic heart disease: the importance and possible ways of correction. Endothelium as a universal regulator of the cardiovascular system. Ukrainian Cardiology Magazine. 2001; 3(1):79-83.

4. Lutai M., Slobodskoy V. Endothelial dysfunction with ischemic heart disease: the importance and possible ways of correction. Endothelial dysfunction is a key link in pathogenesis of cardiovascular pathology and possible ways of its correction (the role of inhibitors of angiotensin converting enzyme). Ukrainian Journal of Cardiology. 2001;4:91-96.

5. Kovaleva 0., Belovol A., Zaika M. The role of oxidative stress in cardiovascular pathology. Journal AMS of Ukraine. 2005; 11(4):660-70.

6. Selivanova G. Cardiovascular manifestations of hypothyroidism: the role and place of hypertension. Cardiologist. 2006;4:64-69.

7. Anderson G., Biakeman N., Streeten D. The effect of age on prevalence of secondary form of hypertension in 4429 consecutively referred patients. J. Hypertens. 1994;12(5):609-15.

8. Dovgan R., Stechenko L., Zagorodniy M., Chekman I. Morphofunctional features of the cardiac myocardial ultrastructure of rats with spontaneous arterial hypertension. Visn. of morphology. 2007;13(2):255-60

9. Kravtsov G., Dumen N., Postnov Yu. Binding of calcium to cytoskeleton of red blood cells in rats with spontaneous hypertension. Cardiology. 1991;10:77-81.

10. Antonov Ye., Alexandrovich Yu., Seryapina A., Klimov L. et al. Stress and arterial hypertension: ISIAH rat strain. Vavilovskii Zhurnal Genetiki i Selektsii -Vavilov Journal of Genetics and Breeding. 2015;19(4):455-59. DOI: 10.18699/VJ15.060

11. Chuhray S., Lavrynenko V., Kaminsky R., Dzevulska I. et al. Morphofunctional status of cardio-vascular system of rats with congenital hypotireosis. Wiad Lek 2019; 72(2):59-63.

12. Chuhray S., Lavrynenko V., Kaminsky R., Ustymenko 0. et al. The influence of drug treatment on cardio-vascular system of the rats with congenital hypothyroidism. Wiad Lek. 2019; 72(7):1300-1303.

13. Chuhray S., Lavrynenko V., Kaminsky R., Dzevulska I. et al. Morphofunctional status of cardio-vascular system of rats with arterial hypertension. Wiad Lek. 2020;73(2):355-359.

14. Chuhray S., Lavrynenko V., Matkivska R., Lachtadyr T. et al. Influence of combined pharmacotherapy on cardio-vascular system of arterial hypertension (in experiment). Wiad Lek. 2020;73(8):1712-1716.

15. Karupu V. Electron microscopy. Vishcha School. 1984: 208.

16. Vaziri N., Rodriguez-Iturbe B. Mechanisms of disease: oxidative stress and inflammation in the pathogenesis of hypertension. Nat. Clin. Pract. Nephrol. 2006; 2(10):582-93. D01:10.1038/ncpneph0283.

17. Wilcox C. Oxidative stress and nitric oxide deficiency in the kidney: a critical link to hypertension? Am. J. Physiol. Regul. Integr. Comp. Physiol. 2005;289(4):913-35. D0I: 10.1152/ajpregu.00250.2005.

18. Kahler J., Ewert A., Weckmuller J., Stobbe S. et al. Oxidative stress increases endothelin-1 synthesis in human coronary artery smooth muscle cells. J. Cardiovasc. Pharmacol. 2001; 38(1):49-57.

19. Touyz R. Reactive oxygen species as mediators of calcium signaling by angiotensin II: implications in vascular physiology and pathophysiology. Antioxid. RedoxSignal.2005:7(9-10):1302-14.D0l:10.1089/ars.2005.7.1302. 
20. Kunsch C., Medford R. Oxidative stress as a regulator of gene expression in the vasculature. Circ. Res.1999; 85(8):753-66.

21. Rodriguez-Iturbe B., Vaziri N., Herrera-Acosta J., Johnson R. Oxidative stress, renal infiltration of immune cells, and salt-sensitive hypertension: all for one and one for all. Am.J.Physiol.Renal Physiol. 2004;286(4):F60616. D0I: 10.1152/ajprenal.00269.2003.

22. Ding L., Jia C., Zhang Y., Wang W. et al. Baicalin relaxes vascular smooth muscle and lowers blood pressure in spontaneously hypertensive rats. Biomedicine \& Pharmacotherapy. 2019;111(3):325-330. D0I: 10.1016/j. biopha.2018.12.086.

23. Papie M., Kaja M., Gêbarowska A. Age-dependent different action of curcumin in thyroid of rat. Folia Histochemica Et Cytobiologica. 2008; 46(2):205-11. doi: 10.2478/v10042-008-0031-6.

The authors would like to thank the employees of the Scientific Research Institute Experimental and Clinical Medicine (Institute of Pathologists Problems) at Bogomolets National Medical University prof. L.O. Stechenko and T.P.Kuftyreva.

\section{ORCID and contributionship:}

Svetlana N. Chuhray: 0000-0001-7431-7375 A,B,C

Viktoria E. Lavrynenko: 0000-0002-2570-1271 ${ }^{\mathrm{F}}$

Rostyslav F. Kaminsky: 0000-0001-5744-7581 ${ }^{\mathrm{C}}$

Larysa B. Shobat: 0000-0002-8592-520X ${ }^{B}$

Oleksandr I. Kovalchuk: 0000-0002-6311-3518 ${ }^{F}$

Yuri B. Chaikovsky: 0000-0002-8821-8930 ${ }^{\mathrm{E}}$

Liudmyla M. Sokurenko: 0000-0002-6870-2290 D,E

\section{Conflict of interest:}

The Authors declare no conflict of interest.

\section{CORRESPONDING AUTHOR}

Liudmyla M. Sokurenko

Bogomolets National Medical University

13 T. Shevchenko st., 01601 Kyiv, Ukraine

tel: +380675010815

e-mail: I-sokurenko@i.ua

Received: 21.11.2019

Accepted: 24.08 .2020

A - Work concept and design, B - Data collection and analysis, C - Responsibility for statistical analysis, D-Writing the article, $\mathbf{E}$-Critical review, $\mathbf{F}$ - Final approval of the article 